\title{
Enmienda con dolomita en la instalación del cultivo de Coffea arábica L. variedad Catuai, en Satipo
}

\section{Amendment with dolomite in the Coffea arabica cultivation L. Catuai variety, in Satipo.}

\author{
Carlos Marcelo O. (1) \\ (1)Facultad de Ciencias Agrarias, Universidad Nacional del Centro del Perú \\ Email: cmarcelo@uncp.edu.pe
}

\section{RESUMEN}

La dolomita es una enmienda muy usada en la agricultura, por su efecto químico en el suelo; por lo que, se planteó el siguiente problema ¿Cuál es el efecto de la aplicación de dolomita en la instalación del cultivo de Coffea arábica $L$. variedad Catuai, en Satipo?, teniendo como objetivos: Evaluar el efecto de la aplicación de dolomita en las características morfológicas y determinar la eficiencia económica de la aplicación de dolomita en el cultivo de Coffea arábica $L$. variedad Catuai. Se trasplantó plantones de café catuai en curvas de nivel, se aplicó dosis de dolomita (0, 200, 400 y $600 \mathrm{Kg} / \mathrm{ha})$. Se evaluó la altura de planta, diámetro de tallo, número de hojas, área foliar y la eficiencia económica de la aplicación de dolomita. Los resultados indican que al aplicar las dosis de $200 \mathrm{Kg} /$ ha de dolomita, las plantas presentan mayor diámetro de tallo y área foliar, ninguna dosis de dolomita mejora el número de hojas, número de ramas y altura de plantas del cafeto a los 9 meses de trasplante. Al comparar la ecuación de predicción del diámetro de tallo y el área foliar, con la pendiente de los costos incurridos, se obtiene que hasta una dosis de 300 kilogramos de dolomita por hectárea, el diámetro de las plantas de café catuai responden económicamente al encalado. Mientras que para el área foliar, en las plantas de café, la respuesta es hasta los 387,5 kilogramos de dolomita por hectárea.

Palabras clave: Enmienda con dolomita - Cultivo de café - Café arábigo - Morfología del cultivo.

\begin{abstract}
Is the dolomita an amendment very used in the agriculture by its chemical effect in the soil for what thought about the following problem Which the effect of the dolomita application is in the installation of the cultivation of Coffea arábica L. variety Catuai, in Satipo?, having as objectives: To evaluate the effect of the dolomita application in the morphological characteristics and to determine the economic efficiency of the dolomita application in the coffe tree variety Catuai. You transplants coffe tree variety catuai in curved of level, dolomita dose was applied (0, 200, 400 and $600 \mathrm{Kg} / \mathrm{ha}$ ). the plant height, shaft diameter, number of leaves was evaluated, area to foliate and you the economic efficiency of the dolomita application. The results indicate that when applying the doses of $200 \mathrm{Kg} / \mathrm{ha}$ of dolomita, the plants present bigger shaft diameter and area to foliate, no dolomita dose improves the number of leaves, number of branches and height of plants from the coffee to the 9 months of transplant. When comparing the equation of prediction of the shaft diameter and area to foliate, with the slope of the incurred costs, it is obtained that until a dose of 300 kilograms of dolomita for hectare the diameter of the plants of coffe tree catuai responds economically to the one whitewashed. While for the area to foliate in the plants of coffee the answer is until the 387,5 kilograms of dolomita for hectare.
\end{abstract}

Key words: Amendment with dolomite - Coffee growing - Arabic coffee - Crop morphology. 


\section{INTRODUCCIÓN}

Los suelos de selva se caracterizan por ser ácidos, siendo la acidez causada por el lavaje de cationes básicos y la presencia de aluminio cambiable. Considerando que la dolomita es fuente de calcio y magnesio, puede ser utilizado como fertilizante para una adecuada nutrición con estos nutrientes. Por lo que, se pretende utilizar diferentes dosis en el cultivo de café y determinar su efecto en el crecimiento de las plantas.

El problema planteado fue ¿Cuál es el efecto de la aplicación de dolomita en la instalación del cultivo de Coffea arábica L. variedad Catuai, en Satipo? Teniendo como objetivos:

- Evaluar el efecto de la aplicación de dolomita en las características morfológicas del cultivo de Coffea arábica L. variedad Catuai.

- Determinar la eficiencia económica de la aplicación de dolomita en las características morfológicas del cultivo de Coffea arábica L. variedad Catuai.

\section{MATERIALES Y MÉTODOS}

El trabajo se realizó en el módulo de café de la Estación Experimental Agropecuaria La Granja.

\section{Población}

La población está constituida por 15 plantas por cada unidad experimental.

\section{Muestra}

La muestra está constituida por 5 plantas por cada unidad experimental.

\section{Procesamiento y análisis de datos}

Para el análisis estadístico se realizó el análisis de varianza al 0,05 y la prueba de comparación de medias (Tukey al 0,05).

\section{Actividades realizadas}

1. Se trasplantó plantones de café catuai en curvas de nivel a 1 metro entre plantas y 2 metros entre filas.

2. Se aplicó dolomita alrededor de las plantas según dosis propuestas.

3. Se realizó las evaluaciones de altura de planta, número de hojas, área foliar y diámetro de tallo, a los 9 meses después de su aplicación.

4. Se refinaron, transformaron los datos y se realizó el análisis estadístico.

\section{RESULTADOS}

Efecto de la aplicación de dolomita en las características morfológicas del cultivo de Coffea arábica $L$. variedad Catuai

Tabla $\mathbf{N}^{\circ}$ 01. Análisis de varianza del efecto de las dosis de dolomita en la altura de las plantas de cafeto, expresados en centímetros

\begin{tabular}{lccccc}
\multicolumn{1}{c}{ Fuente } & $\begin{array}{c}\text { Suma de } \\
\text { cuadrados }\end{array}$ & GI & $\begin{array}{c}\text { Cuadrado } \\
\text { medio }\end{array}$ & Razón-F & Valor-P \\
$\begin{array}{lccccc}\text { Dosis de } \\
\text { dolomita }\end{array}$ & 226,565 & 3 & 75,5217 & 2,48 & 0,1584 \\
Bloque & 131,351 & 2 & 65,6757 & 2,16 & 0,1969 \\
Residuos & 182,688 & 6 & 30,4479 & & \\
Total & $\mathbf{5 4 0 , 6 0 4}$ & $\mathbf{1 1}$ & & & \\
\hline $\mathrm{S}=5,518$ & & & & & \\
\hline
\end{tabular}

En la tabla 01, análisis de varianza del efecto de las dosis de dolomita en la altura de las plantas de cafeto, se observa que entre dosis de dolomita no existe diferencia estadística significativa (Valor - $P$ $0,1584>0,05)$, lo cual indica que las dosis de dolomita no influyeron en la altura de las plantas de cafeto, a los 9 meses.

Entre bloques no existen diferencias estadísticas significativas (Valor -P 0,1969>0,05), lo cual indican, que los bloques no influyeron en la altura de las plantas de cafeto.

El coeficiente de variabilidad de 11,20\% es considerado como bajo, e indica que la altura de las plantas de cafeto dentro de las fuentes de variabilidad es homogéneo.

Tabla $\mathbf{N}^{\circ}$ 02. Análisis de varianza del efecto de las dosis de dolomita en el diámetro del tallo de las

plantas de cafeto, expresados en centímetros

\begin{tabular}{lcccccc}
\multicolumn{1}{c}{ Fuente } & $\begin{array}{c}\text { Suma de } \\
\text { cuadrados }\end{array}$ & GI & $\begin{array}{c}\text { Cuadrado } \\
\text { medio }\end{array}$ & Razón-F & Valor-P \\
$\begin{array}{l}\text { Dosis de } \\
\text { dolomita }\end{array}$ & 0,176692 & 3 & 0,058897 & 6,37 & 0,0270 \\
Bloque & 0,117917 & 2 & 0,058958 & 6,38 & 0,0328 \\
Residuos & 0,055483 & 6 & 0,009247 & & \\
Total & $\mathbf{0 , 3 5 0 0 9 2}$ & $\mathbf{1 1}$ & & & \\
\hline
\end{tabular}

$\mathrm{S}=0,0962 \quad$ Promedio $=1,15 \quad \mathrm{CV}=8,40 \%$

En la tabla 02, análisis de varianza del efecto de las dosis de dolomita en el diámetro del tallo de las plantas de cafeto, se observa que entre dosis de dolomita existen diferencias estadísticas significativas (Valor- $P$ $0,0270<0,05)$, indicando que las dosis de dolomita influye en el diámetro del tallo de las plantas de cafeto, a los 9 meses.

Entre bloques existen diferencias estadísticas significativas (Valor-P 0,0328<0,05), lo que confirma que los bloques influyen en el diámetro del tallo de las plantas de cafeto.

El coeficiente de variabilidad de $8,40 \%$ es considerado como muy bajo, e indica que el diámetro del tallo de las plantas de cafeto dentro de las fuentes de variabilidad es muy homogéneo.

Tabla $N^{\circ}$ 03. Prueba de comparación de medias del efecto de las dosis de dolomita en el diámetro del tallo de las plantas de cafeto, expresados en centímetros 


\begin{tabular}{ccc}
$\begin{array}{c}\text { Dosis de } \\
\text { dolomita }\end{array}$ & Media LS & $\begin{array}{c}\text { Grupos } \\
\text { homogéneos }\end{array}$ \\
\hline 0 & 0,98667 & $\mathrm{a}$ \\
600 & 1,07000 & $\mathrm{a} \mathrm{b}$ \\
400 & 1,25333 & $\mathrm{a} \mathrm{b}$ \\
200 & 1,27333 & $\mathrm{~b}$ \\
& $A L S\left(T_{0,05}\right)=0,270904$ &
\end{tabular}

En la tabla 03, prueba de comparación de medias del efecto de las dosis de dolomita en el diámetro del tallo de las plantas de cafeto, se observa que sin la aplicación de dolomita las plantas presentan menor diámetro de tallo $(0,98$ centímetros $)$ y muestra diferencia estadística significativa con la dosis 200 gramos de dolomita $(1,27$ centímetros). Las plantas encaladas con dosis de 600, 400 y 200 gramos de dolomita no muestran diferencias significativas en el diámetro del tallo de las plantas de cafeto.

Tabla $\mathbf{N}^{\circ}$ 04. Prueba de comparación de medias del efecto de los bloques en el diámetro del tallo de las plantas de cafeto, expresados en centímetros

$\begin{array}{ccc}\begin{array}{c}\text { Dosis de } \\ \text { dolomita }\end{array} & \text { Media LS } & \begin{array}{c}\text { Grupos } \\ \text { homogéneos }\end{array} \\ 1 & 1,0125 & \mathrm{a} \\ 2 & 1,1750 & \mathrm{a} \mathrm{b} \\ 3 & 1,2500 & \mathrm{~b} \\ & \operatorname{ALS}\left(T_{0,05}\right)=0,208635 & \end{array}$

En la tabla 04, prueba de comparación de medias del efecto de bloques en el diámetro del tallo de las plantas de cafeto, se observa que las plantas del bloque 1 presentan menor diámetro de tallo (1,01 centímetros) y muestran diferencias estadísticas significativas con el bloque tres (1,25 centímetros). Las plantas, del bloque dos y tres, no muestran diferencias estadísticas significativas en el diámetro del tallo de las plantas de cafeto.

Tabla $\mathbf{N}^{\circ}$ 05. Análisis de varianza del efecto de las dosis de dolomita en el número de hojas de las plantas de cafeto, expresados en centímetros cuadrados

\begin{tabular}{lccccc}
\multicolumn{1}{c}{ Fuente } & $\begin{array}{c}\text { Suma de } \\
\text { cuadrados }\end{array}$ & GI & $\begin{array}{c}\text { Cuadrado } \\
\text { medio }\end{array}$ & Razón-F & Valor-P \\
\hline $\begin{array}{l}\text { Dosis de } \\
\text { dolomita }\end{array}$ & 4,75389 & 3 & 1,584630 & 3,19 & 0,1055 \\
Bloque & 0,700867 & 2 & 0,350433 & 0,71 & 0,5308 \\
Residuos & 2,98153 & 6 & 0,496922 & & \\
Total & $\mathbf{8 , 4 3 6 2 9}$ & $\mathbf{1 1}$ & & & \\
$S=0,7049$ & \multicolumn{2}{c}{ Promedio $=9,08$} & $C V=7,77 \%$
\end{tabular}

En la tabla 05, análisis de varianza del efecto de las dosis de dolomita el número de hojas de las plantas de cafeto, se observa que entre dosis de dolomita no existen diferencias estadísticas significativas (Valor-P 0,1055>0,05), indicando que las dosis de dolomita no influyeron en el número de hojas de las plantas de cafeto, a los 9 meses.

Entre bloques no existen diferencias estadísticas significativas (Valor -P 0,5308>0,05), lo que determina que los bloques no influyeron en el número de hojas de las plantas de cafeto.

El coeficiente de variabilidad de 7,77\% es considerado como muy bajo, e indica que el número de hojas de las plantas de cafeto dentro de las fuentes de variabilidad es muy homogéneo.

Tabla $\mathbf{N}^{\circ}$ 06. Análisis de varianza del efecto de las dosis de dolomita en el área foliar de las plantas de cafeto, expresados en centímetros cuadrados

\begin{tabular}{lcccccc}
\multicolumn{1}{c}{ Fuente } & $\begin{array}{c}\text { Suma de } \\
\text { cuadrados }\end{array}$ & Gl & $\begin{array}{c}\text { Cuadrado } \\
\text { medio }\end{array}$ & Razón-F & Valor-P \\
\hline $\begin{array}{l}\text { Dosis de } \\
\text { dolomita }\end{array}$ & 302,311 & 3 & 100,770 & 7,05 & 0,0216 \\
Bloque & 11,5232 & 2 & 5,76161 & 0,40 & 0,6851 \\
Residuos & 85,7599 & 6 & 14,2933 & & \\
Total & $\mathbf{3 9 9 , 5 9 4 2}$ & $\mathbf{1 1}$ & & \\
$S=3,7806$ & \multicolumn{5}{c}{ Promedio $=41,79$} & $C V=9,05 \%$
\end{tabular}

En la tabla 06, análisis de varianza del efecto de las dosis de dolomita en el área foliar de las plantas de cafeto, se observa que entre dosis de dolomita existen diferencias estadísticas significativas (Valor -P 0,0216<0,05), evidenciando que las dosis de dolomita influyen en el área foliar de las plantas de cafeto, a los 9 meses.

Entre bloques no existen diferencias estadísticas significativas (Valor -P 0,6851>05), señalando que los bloques no influyen en área foliar de las plantas de cafeto.

El coeficiente de variabilidad de 9,05\% es considerado como muy bajo, e indica que el área foliar de las plantas de cafeto dentro de las fuentes de variabilidad es muy homogéneo.

Tabla $\mathbf{N}^{\circ}$ 07. Prueba de comparación de medias del efecto de las dosis de dolomita en el área foliar de las plantas de cafeto, expresados en centímetros cuadrados

$\begin{array}{ccc}\begin{array}{c}\text { Dosis de } \\ \text { dolomita }\end{array} & \text { Media LS } & \begin{array}{c}\text { Grupos } \\ \text { homogéneos }\end{array} \\ 0 & 35,3400 & \mathrm{a} \\ 600 & 38,4600 & \mathrm{a} \mathrm{b} \\ 400 & 46,2767 & \mathrm{~b} \\ 200 & 47,0767 & \mathrm{~b} \\ & A L S\left(T_{0,05}\right)=10,6506 & \end{array}$

En la tabla 07, prueba de comparación de medias del efecto de las dosis de dolomita en el área foliar de las plantas de cafeto, se observa que las plantas, sin la aplicación de dolomita, presentan menor área foliar $(35,34$ centímetros cuadrados) y muestran diferencias estadísticas significativas con las plantas fertilizadas con dosis $400 \mathrm{y}$ 
200 gramos de dolomita que presentan hojas con 46,27 y 47,07 centímetros cuadrados. Las plantas encaladas con dosis de 600, 400 y 200 gramos de dolomita no muestran diferencias en el área foliar, entre ellas.

Tabla $\mathbf{N}^{\circ}$ 08. Análisis de varianza del efecto

de las dosis de dolomita el número de ramas de las plantas de cafeto, expresados en centímetros cuadrados

\begin{tabular}{lcccccc|}
\multicolumn{1}{c}{ Fuente } & $\begin{array}{c}\text { Suma de } \\
\text { Cuadrados }\end{array}$ & Gl & $\begin{array}{c}\text { Cuadrado } \\
\text { Medio }\end{array}$ & Razón-F & Valor-P \\
\hline $\begin{array}{l}\text { Dosis de } \\
\text { dolomita }\end{array}$ & 0,511492 & 3 & 0,170497 & 3,24 & 0,1028 \\
Bloque & 0,339217 & 2 & 0,169608 & 3,22 & 0,1122 \\
Residuos & 0,315983 & 6 & 0,052664 & & \\
\hline Total & $\mathbf{1 , 1 6 6 6 9 2}$ & $\mathbf{1 1}$ & & & \\
\hline S $=0,2295$ & \multicolumn{2}{c}{ Promedio $=3,52$} & & CV $=6,53 \%$
\end{tabular}

En la tabla 08, análisis de varianza del efecto de las dosis de dolomita en el número de ramas de las plantas del cafeto, se observa que entre dosis de dolomita no existen diferencias estadísticas significativas (Valor-P 0,1028>0,05), denota que, las dosis de dolomita, no influyeron en el número de ramas de las plantas del cafeto, a los 9 meses.

Entre bloques no existe diferencia estadística significativa (Valor-P 0,1122>0,05), demostrando que los bloques no influyeron en el número de ramas de las plantas del cafeto.

El coeficiente de variabilidad de 6,53\% es considerado como muy bajo, e indica que el número de ramas de las plantas de cafeto dentro de las fuentes de variabilidad en muy homogéneo.

Eficiencia económica de la aplicación de dolomita en las características morfológicas del cultivo de Coffea arábica L. variedad Catuai

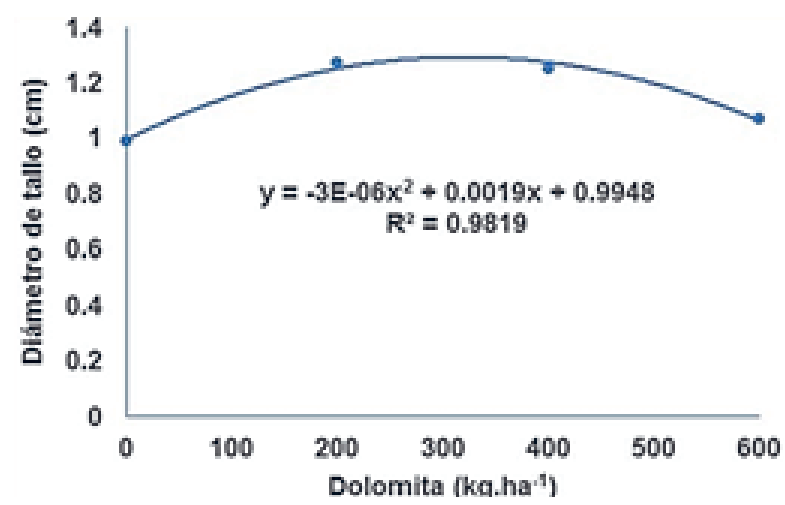

Figura 01. Efecto de las dosis de dolomita en el diámetro de tallo del cafeto, a los 9 meses

En la figua 01, se observa que al incrementar las dosis de dolomita, se incrementa el diámetro de tallo, al superar la dosis de 316.67 kilogramos de dolomita por hectárea el diámetro de las plantas de café catuai tienden a disminuir.

- Ecuación 1: Dosis máxima de dolomita en el diámetro de tallo

$$
\begin{gathered}
0=(2 *-0.000003) X+0.0019 \\
X=\frac{0.000006 X=0.0019}{0.000006}=316.67
\end{gathered}
$$

Al comparar con la pendiente de los costos incurridos por el incremento de las dosis de dolomita, se obtiene que hasta una dosis de 300 kilogramos de dolomita por hectárea, el diámetro de las plantas de café catuai responden económicamente al encalado con dolomita.

- Ecuación 2: Dosis máxima rentable de dolomita en el diámetro de tallo

$$
0.0001=(2 *-0.000003) X+0.0019
$$

$$
0.000006 X=0.0019-0.0001
$$

$$
x=\frac{0.018}{0.000006}=300
$$

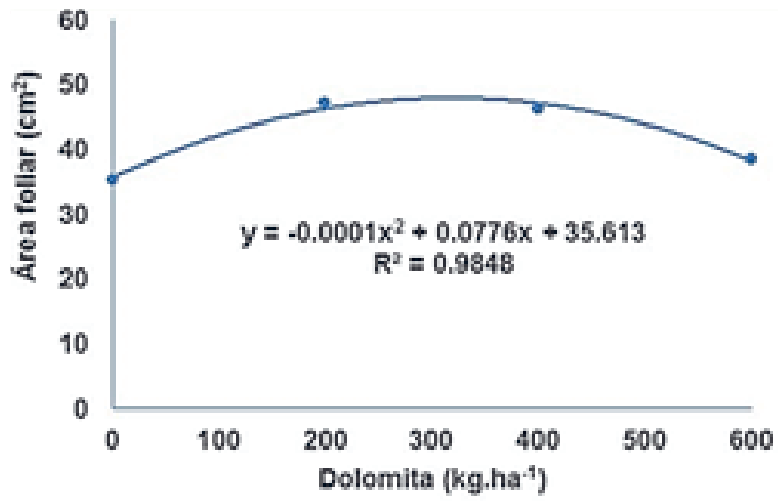

Figura 2. Efecto de las dosis de dolomita en el área foliar de las hojas del cafeto, a los 9 meses

En el grafico 02, se observa que al incrementar las dosis de dolomita, se incrementa el área foliar en las plantas de café, al superar la dosis de 388 kilogramos de dolomita por hectárea el área foliar en las plantas de café catuai tienden a disminuir.

- Ecuación 3: Dosis máxima de dolomita en el área foliar

$$
\begin{gathered}
0=(2 *-0.0001) X+0.0776 \\
0.0002 X=0.0776 \\
X=\frac{0.0776}{0.0002}=388
\end{gathered}
$$

Al comparar con la pendiente de los costos incurridos por el incremento de las dosis de dolomita, se obtiene hasta 387,5 kilogramos de dolomita por hectárea. El área foliar en las plantas de café catuai responden económicamente al encalado con dolomita.

- Ecuación 2: Dosis máxima rentable de dolomita en el diámetro de en el área foliar 
$0.0001=(2 *-0.0001) X+0.0776$

$0.0002 X=0.0776-0.001$

$$
x=\frac{0.0745}{0.0002}=387.5
$$

\section{Discusión}

Del efecto de la aplicación de dolomita en las características morfológicas del cultivo de $\mathrm{Co}$ ffea arábica L. variedad Catuai

La aplicación de dosis de dolomita, tiene efecto en el diámetro del tallo y en el área foliar de las plantas de cafeto. Las plantas encaladas, con dosis 400 y 200 gramos de dolomita, presentan hojas con 46,27 y 47,07 centímetros cuadrados, superiores a las plantas sin encalar. Esto indica que se incrementan las células del tallo y las hojas de las plantas de café por efecto del calcio y magnesio aplicados con la dolomita. La dolomita al contener calcio y magnesio provee de mayor cantidad de estos elementos a la planta, tal como lo indica Molina (1998. p.5), las sales básicas que contienen calcio y magnesio son muy abundantes en la naturaleza, y estos dos nutrientes son esenciales para las plantas. Asimismo, Durán (2007), indica que el encalado es la técnica usada para aportar nutrientes, como el calcio y el magnesio.

El crecimiento, del tallo y hojas, se debe al calcio que hace las paredes de la célula más fuertes porque forma parte de los pectato de calcio y juega un papel en la formación de proteína y el movimiento de hidratos de carbono en las plantas (Plaster, 2000). Por su parte Thompson y Troeh (1988, p. 392) confirman que el calcio forma parte de la pared celular, por lo que es vital para la formación de nueva células.

Los resultados del análisis de suelo reportan un contenido de calcio extractable muy bajo $(0,5 \mathrm{meq} / 100 \mathrm{~g})$, y un contenido de magnesio extractable bajo $(0,65$ meq $/ 100 \mathrm{~g})$ y por su parte Thompson y Troeh $(1988$, p. 393) coinciden en que la provisión de calcio es menor en suelos ácidos y hace más peligrosa la toxicidad del aluminio. También afirman que las concentraciones más bajas de calcio ocurren en suelos lavados del trópico. En suelos pueden responder a la fertilización con calcio (p. 390).

Además, al aplicar dolomita también se adiciona magnesio que es móvil en la planta y es vital para la producción de clorofila y su función fotosintética. La mayor parte del magnesio se encuentra en la clorofila y las semillas, una parte funciona en el sistema enzimático de los carbohidratos (Idem, 1988, p. 396). Por tanto, La mayor parte del magnesio aplicado proviene de la dolomita, su deficiencia en el suelo se debe a la elevada precipitación que lava el magnesio, ocurriendo en zonas tropicales como Satipo.
Rojas (1993, p. 149) reporta que la variación en el área foliar es el principal factor que determina diferencias en el rendimiento, por lo que al incrementarse el área foliar de las plantas, se espera tener una mayor producción de granos de café al encalar con dolomita. Aunque Braeuner, Ortiz, y MacVean (2005. p. 17-24) reportaron que ante el incremento de calcio y magnesio, no hubo diferencias en la producción de grano de café.

De la eficiencia económica de la aplicación de dolomita en las características morfológicas del cultivo de Coffea arábica $L$. variedad Catuai

Al comparar con la pendiente de los costos incurridos por el incremento de las dosis de dolomita, se obtiene que hasta una dosis de 300 kilogramos de dolomita por hectárea el diámetro de las plantas de café catuai responden económicamente al encalado con dolomita. Mientras que para el área foliar en las plantas de café la respuesta es hasta los 387,5 kilogramos de dolomita por hectárea.

El incremento inicial se debe a que el suelo presenta un contenido bajo de calcio y magnesio, al encalar se suplementa los requerimientos del cultivo, tal como lo menciona Molina (1998). La deficiencia se supera hasta los 300 y 387,5 kilogramos de dolomita por hectárea, en relación al diámetro y área foliar.

Cantidades superiores tienen un efecto negativo, se pasa a la zona donde la pendiente es negativa, comparando con la pendiente de los costos de las dosis de dolomita. Esto se debería a que un exceso de calcio en el suelo limita la solubilidad del fósforo, hierro, manganeso, boro y zinc, causando su deficiencia de estos nutrientes para las plantas (Thompson y Troeh, 1988, p. 391).

En referencia a ley de asimilación decreciente, Barceló, Nicolás, Sabater y Sánchez (2001, p. 126) recomiendan que, "Cuanto más se acerque la curva a la fase óptima menos eficaz será el aporte mineral desde el punto de vista del crecimiento".

\section{REFERENCIAS BIBLIOGRÁFICAS}

Barceló, J.; Nicolás, G.; Sabater, B. y Sánchez R. 2001. Fisiología vegetal. Ediciones Pirámide. Madrid.

Braeuner, M.; Ortiz, R. y MacVean, Ch. 2005. Efectos de la aplicación de cal dolomítica y yeso agrícola en cafetales (Coffea arabica) afectados con mal de Viñas en Guatemala. Manejo integrado de plagas y agroecología (Costa Rica) No. 76. p. 17-24.

Durán, F. 2007. Cultivo del café. Grupo Latino Editores S.A.S. Colombia.

Mejía, S. y Cahuapaza, H. 2001. Sistemas de producción y manejo del café. Conservación Internacional. 
Molina, E. 1988. Acidez de suelo y encalado. Centro de Investigaciones Agronómicas. Universidad de Costa Rica.

Ortiz, E. 2008. Evaluación del efecto de la cal dolomita sobre algunas características químicas del suelo y la absorción de nutrientes en el cultivo de piña (Ananas comosus) (l) merr. Híbrido md-2 en finca ganadera la Flor S.A. en Río Cuarto, Grecia, Costa Rica.Instituto tecnológico de costa rica. San Carlos, Costa Rica.

Plaster, E. 2000. La ciencia del suelo y su manejo. Madrid: Thomson Editores Spain.

Tisdale, S. y Nelson, W. 1977. Fertilidad de los Suelos y fertilizantes. Barcelona: Montaner y Simón.

Thompson, L y Troeh, F. 1988. Los suelos y su fertilidad. Editorial Reverté. Barcelona.

La Fundación para el Desarrollo Socio Económico y Restauración Ambiental (FUNDESyRAM). 2010. Guía para la innovación de la caficultura. De lo convencional a lo orgánico. FUNDESyRAM. San Salvador.

Durán, F. 2007. Cultivo del café. Grupo Latino Editores S.A.S. Colombia.

Molina, E. 1998. Encalado para la corrección de la acidez del suelo, ACCS, San José, Costa Rica. 45 p. 